\title{
Aa. Vv., «George Sand Studies»
}

\section{Morena Petrich}

\section{(2) OpenEdition}

\section{Journals}

\section{Edizione digitale}

URL: http://journals.openedition.org/studifrancesi/8313

DOI: 10.4000/studifrancesi.8313

ISSN: 2421-5856

\section{Editore}

Rosenberg \& Sellier

\section{Edizione cartacea}

Data di pubblicazione: 1 mai 2009

Paginazione: 195-197

ISSN: 0039-2944

\section{Notizia bibliografica digitale}

Morena Petrich, «Aa. VV., «George Sand Studies»», Studi Francesi [Online], 157 (LIII | I) | 2009, online dal 30 novembre 2015, consultato il 08 janvier 2021. URL: http://journals.openedition.org/studifrancesi/ 8313 ; DOI: https://doi.org/10.4000/studifrancesi.8313

Questo documento è stato generato automaticamente il 8 janvier 2021.

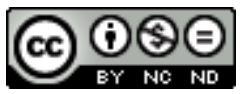

Studi Francesi è distribuita con Licenza Creative Commons Attribuzione - Non commerciale - Non opere derivate 4.0 Internazionale. 


\section{Aa. Vv., «George Sand Studies»}

\section{Morena Petrich}

\section{NOTIZIA}

«George Sand Studies», t. XXVI, 2007, pp. 115.

1 La rivista si apre con un interessante contributo di James F. HAMILTON («Sand's La Mare au diable, the "Appendice" as Peasant Ritual and Narrative Closure», pp. 3-14), che ricolloca debitamente in un ruolo più pregnante che accessorio l'appendice del romanzo di Sand del 1846. La marcata rispondenza speculare tra la digressione iniziale e la fantaisie conclusiva sottolinea la forte valenza narrativa della glossa finale mentre i metatesti che compaiono con frequenza e connotano segnatamente le pagine di La Mare au diable evidenziano un fitto simbolismo teso a delineare l'evoluzione nello scritto sandiano dall'individualismo militante dell'esordio al bene collettivo dell'epilogo. La complessità simbolica del contenuto del romanzo è presa in esame dal punto di vista della valenza sessuata, sotto diverse angolature: la solennità e la conservazione del lignaggio matrilineare; il nesso soggiacente tra violenza e sessualità; la ritualità e lo statuto matrimoniale in contesto mitico-arcaico. È partendo da una riflessione sulla parolachiave «bête», così frequente nelle opere di Sand così come nei commenti a lei stessa rivolti da alcuni illustri colleghi, che prende le mosse la ricca analisi di Marie-Christine GARNEAU DE L'ISLE-ADAMsulla componente bucolica negli scritti dell'autrice (Du côté de chez Virgile, à l'ombre des "Géorgiques" en fleurs, pp. 15-27). Il mondo campestre dei romanzi sandiani riecheggia i grandi autori della tradizione classica, in particolare Virgilio e Teocrito, ma anche Omero. Iniziata allo studio del latino e all'epica sin dagli studi giovanili, l'autrice farà rivivere sempre più nei suoi testi la "poétique de la terre» poiché, come mette in evidenza l'autore, il contesto rustico e bucolico, proprio per l'insita celebrazione di un'età aurea, rappresenta uno sfondo privilegiato per il messaggio profetico sandiano, notoriamente repubblicano e cristiano-evangelico al contempo. Attraverso una galleria di personaggi artigiani, artisti o semplici lavoratori modestamente laboriosi, viene illuminato un microcosmo in cui l'utopia umanista approda al popolare, dopo gli esordi aristocratici dei primi scritti e dei loro 
protagonisti. Anche un tema di matrice pitagorica come la riflessione sull'esistenza dell'anima negli animali riaffiora nelle opere della romanziera, in cui il mondo animale è messo in relazione con la sfera istintuale e l'erotismo e in cui la violenza predatorepreda è superata nell'idillio simbiotico pastorale. D'altronde, Sand e Virgilio avevano ricreato l'«utopie sociale champêtre» nei loro rispettivi rifugi dorati, caratterizzati da una forte componente etica e da un rifiuto della corruzione della vita cittadina e della mondanità: l'approccio metafisico nel culto della propria intimità si esprime pienamente nel giardino chiuso degli happy few.

2 Il parallelo tracciato tra le due artiste in La voix perdue de George Sand et Marceline Desbordes-Valmore (pp. 28-42) anima il successivo contributo, di Monia KALLEL. Molte sono le potenziali affinità elettive che avrebbero potuto legare i percorsi e le vite delle due donne: stessa carriera, frequentazioni comuni (tra cui soprattutto quella con il letterato Henri de Latouche), uguale passione per la musica, un breve e in realtà solo formale scambio epistolare. Nonostante tali convergenze il loro rapporto si manterrà sul crinale di una scarsa confidenza se non di una certa, dissimulata, riottosità. L'afasia si delinea come elemento ricorrente negli scritti di entrambe, non solo come vestigio doloroso di ogni atto scritturale ma piuttosto come latenza di percorsi emozionali e moti pulsionali inespressi. Il silenzio della parola si avvicenda e struttura l'alternanza con la musica, che restituisce al soggetto pienezza ed armonia con il tutto. L'autore evidenzia come i temi della musica e della voce siano al centro della poetica sia di Desbordes-Valmore che di Sand e quanto la sintonia dell'individuo con il creato funga da stimolo e supporto alla memoria. La parola assurge nella trama narrativa sandiana e nella lirica valmoriana ad un duplice statuto, portatrice al contempo di una forma «préverbale» e fluida e di un'altra forma più compiuta, propriamente verbalizzata. Nell'oscillazione tra esplicito ed implicito, così come tra comunicazione e silenzio, la perdita della voce travalica la funzione difensiva ed autodifensiva per elevarsi a poetica, che si allontana dall'espressione spontanea del pensiero per approdare ad un messaggio elaborato, strutturato, pienamente letterario. Lo studio di Kyoko MURATA, Sous le signe des monstruosités: la Camille Maupin de Balzac et George Sand (pp. 43-54), mette in luce il pensiero dell'autore de La Comédie humaine sull'amica scrittrice, tracciandone un ritratto che risulta in filigrana dall'analisi del personaggio Camille Maupin, maschera della romanziera. Al di là delle somiglianze riscontrabili dal punto di vista fisico, sono i tratti salienti delle due personalità a delineare l'estensione e l'intensità della congruenza tra i due profili: la mascolinità, le opinioni riguardo la condizione femminile e le relative critiche al vincolo matrimoniale, per com'era allora concepito e codificato dalla legislazione e dai costumi. La condanna sociale che grava sulla protagonista balzachiana ricalca i capi d'accusa mossi a G. Sand stessa: la pratica della scrittura, le avventurose vicissitudini sentimentali, i vizi come il fumo, ma anche le doti intellettuali. L'eccezionalità è percepita dunque come mostruosità ed interpretata come un pericoloso attentato all'ordine patriarcale, alla sua conservazione e alla sua immobilità; si tratta di minacce che, alternandosi agli elogi e alla sincera ammirazione di Balzac per la scrittrice, fanno emergere le debolezze e le contraddizioni di un pensiero concettualmente solidale col genere femminile e la sua necessaria emancipazione, ma ancora parzialmente aderente ai dettami della morale comune. È ancora sull'età dell'oro, questa volta celebrata in particolare per la sua «Sancta Simplicitas», che ritorna l'articolo di Marianne LORENZI (George Sand, 1832-1842. Une esthétique de la simplicité, pp. 55-72). Come dichiara nell'incipit l'autore, il contributo trae spunto da una nota di René Bourgeois nella sua edizione del Compagnon du Tour de 
France, in cui questi sottolinea «la centralità nel testo della simplicité»: lo studio indaga i significati e le valenze del concetto all'interno della produzione sandiana, con riferimento al decennio 1832-1842. La traiettoria evidenziata si snoda da una «semplicità iniziale» ad una «progressiva semplificazione del reale», altamente funzionale alla veicolazione del messaggio ideologico della scrittrice. Alla magniloquenza della forma privilegiata dai suoi contemporanei e alla "surenchère romantique», Sand oppone la sobria ma sostanziale ricchezza dei tempi antichi. In tale prospettiva, Pierre, il protagonista de Le Compagnon (che giustamente "possède la sublime simplicité de l'ouvrier»), con la sua crescita esistenziale incarna perfettamente l'«utopia sociale», sandiana, espressa in una lingua pura ed esemplare, proprio perché genuina. Infine, l'analisi mette in luce quanto la struttura e le potenzialità della parabola siano appropriate a tale tensione, estetica e ideologica e quanto queste coincidano con la semplificazione, adottata come principio compositivo e «modalité du procédé argumentatif».

3 L'epilogo di Mauprat rivela, secondo l'interessante lettura che ne propone Elena PATRICK (George Sand's "Mauprat": A Gendered Way from Aristocracy to Bourgeoisie», pp. 73-87), le ansie della società francese dei turbolenti avvenimenti pre e post-rivoluzionari nei confronti della bourgeoisification, che si staglia sullo sfondo del romanzo in questione. L'autore sottolinea come l'ambiguità identitaria del narratore non avrebbe in realtà potuto nascondere una voce femminile perché la sua funzione e le implicazioni del suo ruolo avrebbero forzatamente richiesto un'identità maschile. Il décor della vicenda è profondamente permeato dalla paura e dal clima di pesante incertezza vissuto dall'aristocrazia, che, uscita sconfitta dallo snodo epocale del '89, come dimostrano le vicende dei protagonisti, mal governa, nella sua difficile e sofferta ricerca di collocazione nel nuovo assetto borghese, anche i conflitti di generi ad essa interni.

Nel suo contributo sulla bibliografia delle pubblicazioni dedicate a George Sand nella stampa berrichonne dal 1833 al 1876, Claire LE GUILLOU mette puntualmente in luce come, nonostante l'esiguità delle pubblicazioni apparse nella regione nell'arco temporale considerato, la presenza dell'autore. si riveli una presenza costante. Ben lungi dal consacrare un'intera pagina alla cultura o, nello specifico, alla letteratura, la stampa regionale (che è spesso costituita da "Affiches", ovvero annunci di natura giudiziaria o notarile) si limita nel periodo in esame ad una sessantina di articoli inerenti la scrittrice, di cui il venti per cento si incentra sulla sua produzione romanzesca (annunci pubblicitari, omaggi e critiche), mentre le restanti note riguardano l'esistenza stessa dell'autrice, la sua attività politica, le pièces teatrali e le loro rappresentazioni. Ci sembra degno di citazione e opportuna chiosa per la nostra rassegna il secondo hommage recensito, che risale al gennaio del 1838 e che, dopo una magniloquente invocazione - «George Sand! Ô grand roi!» - recita così: «Oui, je l'espère, George Sand, un jour viendra où sur le linceul des préjugés s'élèvera le monde de tes pensées. Alors plus de doute, plus d'envie, plus d'ignorance, à la mer battue par le flux et le reflux des idées, aura succédé la mer calme et tranquille, et l'étoile de George Sand servira dès lors de guide à toutes les générations naissantes et à naître». 